R. MIYAOKA

KODAI MATH. J.

12 (1989), 308-315

\title{
DUPIN HYPERSURFACES WITH SIX PRINCIPAL CURVATURES
}

\author{
By Reiko MiYaoka
}

In this paper, we give a necessary and sufficient condition for a compact embedded Dupin hypersurface with six principal curvatures to be Lie equivalent to an isoparametric hypersurface. The argument goes almost parallel with the case of four principal curvatures [1], and we assume all the results contained there, as well as its notations.

Now we state our result.

THEOREM. Let $M$ be a compact embedded Dupin hypersurface in a space form $\bar{M}(c)$. If $M$ has six principal curvatures $\lambda_{1}>\lambda_{2}>\cdots>\lambda_{6}$ at each point of $M$, then $M$ is the Lie-geometric image of an isoparametric hypersurface in a sphere if and only if the following are satisfied.

(i) All funcitons

$$
\Psi_{\imath j k l}:=\left[\lambda_{\imath}, \lambda_{j} ; \lambda_{k}, \lambda_{l}\right]=\frac{\left(\lambda_{i}-\lambda_{k}\right)\left(\lambda_{j}-\lambda_{l}\right)}{\left(\lambda_{i}-\lambda_{l}\right)\left(\lambda_{j}-\lambda_{k}\right)}
$$

are constant on $M$, where $i, j, k, l \in\{1,2, \cdots, 6\}$ are mutually distinct numbers. (ii) For each $\lambda_{1}$-leaf $L^{1}$, there are $\lambda_{3}$-leaf $L_{1}^{3}$ and $\lambda_{5}$-leaf $L_{1}^{5}$ such that $L_{q}^{2} \cap L_{1}^{3} \neq \varnothing$ and $L_{q}^{4} \cap L_{1}^{5} \neq \varnothing$ for all $q \in L^{1}$, where $L_{q}^{2}$ and $L_{q}^{4}$ denote $\lambda_{2}$-leaf and $\lambda_{4}$-leaf at $q$, respectively.

By an elementary calculation, we obtain

LEMMA. Let $\Im_{6}$ be the symmetric group of degree 6 . Then all $\Psi_{\imath j k l}$ 's are constant if and only if

$$
\Psi_{\sigma(1) \sigma(2) \sigma(3) \sigma(4)}, \Psi_{\sigma(1) \sigma(2) \sigma(3) \sigma(5)}, \Psi_{\sigma(1) \sigma(2) \sigma(3) \sigma(6)}
$$

are constant on $M$ for some $\sigma \in \mathbb{S}_{6}$.

Therefore, we can replace (i) by (i)' in the statement of the theorem. This lemma is implied without calculation if we note that the curvature spheres correspond to projective points on the projective line obtained by the Legendre map, and that for fixed three points on the line, the fourth point is determined by the cross ratio (Remark 4.8 [1]).

Received June 8, 1988. 
For the proof of the theorem, necessity (i) is already shown in Corollary of [1], and (ii) will be proved in Proposition 5. To prove sufficiency, we briefly follow $\S 5-9$ of [1], adding some remarks.

This time, we denote six principal curvatures by $\lambda>\mu>\nu>\rho>\sigma>\tau$, and the corresponding orthonormal frame by $\left(e_{a}, e_{f}, e_{\imath}, e_{r}, e_{u}, e_{x}\right)$, where the indices range so that $\left\{e_{a}\right\}=$ the principal distribution with respect to $\lambda$, and so forth. Actually, each distribution is of dimension $m=\frac{n-1}{6}$, by the same argument as in [2]. Under the assumption (i) or (i)', every point of $M$ is a critical point of all $\Psi$ 's. So we get

LEMMA 1 (cf. Lemma 5.3 in [1]). At every point of $M$, we have

$$
\begin{aligned}
\frac{\Lambda_{f f}^{a}-\Lambda_{i i}^{a}}{\mu-\nu} & =\frac{\Lambda_{f f}^{a}-\Lambda_{r r}^{a}}{\mu-\rho}=\frac{\Lambda_{f f}^{a}-\Lambda_{u u}^{a}}{\mu-\sigma}=\frac{\Lambda_{f f}^{a}-\Lambda_{x x}^{a}}{\mu-\tau} \\
& =\frac{\Lambda_{i i}^{a}-\Lambda_{r r}^{a}}{\nu-\rho}=\frac{\Lambda_{i i}^{a}-\Lambda_{u u}^{a}}{\nu-\sigma}=\frac{\Lambda_{i i}^{a}-\Lambda_{x x}^{a}}{\nu-\tau} \\
& =\frac{\Lambda_{r r}^{a}-\Lambda_{u u}^{a}}{\rho-\sigma}=\frac{\Lambda_{r r}^{a}-\Lambda_{x x}^{a}}{\rho-\tau}=\frac{\Lambda_{u u}^{a}-\Lambda_{x x}^{a}}{\sigma-\tau}\left(:=R_{a}\right) .
\end{aligned}
$$

We define $R_{f}, R_{\imath}, R_{r}, R_{u}, R_{x}$ similarly by the corresponding ratios.

Proof. For instance, $e_{a}(\log [\lambda, \rho ; \mu, \nu])=0$ implies

$$
\frac{\Lambda_{f f}^{a}-\Lambda_{r r}^{a}}{\mu-\rho}=\frac{\Lambda_{i i}^{a}-\Lambda_{r r}^{a}}{\nu-\rho}(=: R)
$$

and $e_{a}(\log [\lambda, \sigma ; \mu, \nu])=0$ implies

$$
\frac{\Lambda_{f f}^{a}-\Lambda_{u u}^{a}}{\mu-\sigma}=\frac{\Lambda_{i i}^{a}-\Lambda_{u u}^{a}}{\nu-\sigma}\left(=: R^{\prime}\right) .
$$

Expressing the numerators by $R\left(R^{\prime}\right)$ and denominators, $R=R^{\prime}$ is easily shown.

q.e.d.

LEMMA 2 (cf. Lemma 5.4 of [1]). At a fixed point $p$ of $M$, we obtain $A_{1} \in O(n+1,2)$ such that

$$
\tilde{\Lambda}_{\beta \beta}^{\alpha}(p)=\tilde{\Lambda}_{r r}^{\alpha}(p) \text { for all } \alpha, \beta, \gamma \text { such that } \alpha \notin[\beta] \cup[\gamma], \beta \notin[\gamma] \text {. }
$$

Proof. If we put $b=0$ in (4.7) of $[1],\left(x_{a}, y_{a}\right)=\left(0, d R_{a}\right)$ is a solution of the simultaneous equation

$$
\tilde{\Lambda}_{f f}^{a}(p)=\tilde{\Lambda}_{i i}^{a}(p)=\tilde{\Lambda}_{r r}^{a}(p)=\tilde{\Lambda}_{u u}^{a}(p)=\tilde{\Lambda}_{x x}^{a}(p) .
$$

Then $A_{1}$ is obtained in the same way as the proof of Lemma 5.4. q.e.d.

Now, by Remark 5.5 of $[1]$, we can find $A_{2} \in O(n+1,2)$ so that at the 
image point of $p$ by $A_{2} \circ A_{1}$, the normal geodesic becomes "common".

Denoting the image of $M$ under $A_{2} \circ A_{1}$ by the same letter, we get

Lemma 3 (cf. Proposition 6.1 of [1]). The normal geodesic $\gamma$ at $p$ cuts $M$ at twelve points $p_{1}=p, p_{2}, \cdots, p_{12}$. Moreover, $\gamma$ is the common normal geodesic at every point $p_{i}$, and all leaves at $p_{i}$ 's are connected as in Figure 1.

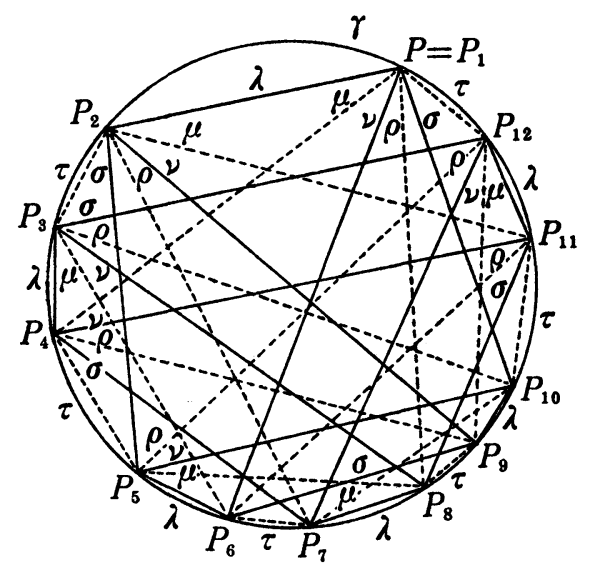

Fig. 1 .

Proof. Let $p_{1}=p, L_{p}^{\lambda} \cap \gamma=\left\{p_{1}, p_{2}\right\}, L_{p}^{\mu} \cap \gamma=\left\{p_{1}, p_{4}\right\}, L_{p}^{\nu} \cap \gamma=\left\{p_{1}, p_{6}\right\}, L_{p}^{\rho} \cap \gamma$ $=\left\{p_{1}, p_{8}\right\}, L_{p}^{\sigma} \cap \gamma=\left[p_{1}, p_{10}\right\}$ and $L_{p}^{\tau} \cap \gamma=\left\{p_{1}, p_{12}\right\}$. Then there exist $p_{3} \in \overparen{p_{2} p_{4}} \cap M$, $p_{5} \in \overparen{p_{4} p_{6}} \cap M, p_{7} \in \overparen{p_{6} p_{8}} \cap M, p_{9} \in \overparen{p_{8} p_{10}} \cap M$ and $p_{11} \in \overparen{p_{10} p_{12}} \cap M$, since $M$ devides $S^{n}$ into two disk bundles over two focal submanifolds consisting of the first focal points of $M$ in both directions (see the proof of Proposition 6.1 in [1]).

We denote homology cycles of $M$ at $p \in M$ obtained by Thorbergsson [3] by

$$
\begin{aligned}
& {\left[c_{p}^{\lambda}\right],\left[c_{p}^{\tau}\right] \in H_{m}\left(M ; \boldsymbol{Z}_{2}\right),} \\
& {\left[c_{p}^{\mu \lambda}\right],\left[c_{p}^{\sigma \tau}\right] \in H_{2 m}\left(M ; \boldsymbol{Z}_{2}\right),} \\
& {\left[c_{p}^{\nu \mu \lambda}\right],\left[c_{p}^{\rho \sigma \tau}\right] \in H_{3 m}\left(M ; \boldsymbol{Z}_{2}\right),} \\
& {\left[c_{p}^{\rho \nu \mu \lambda}\right],\left[c_{p}^{\nu \rho \sigma \tau}\right] \in H_{4 m}\left(M ; \boldsymbol{Z}_{2}\right),} \\
& {\left[c_{p}^{\sigma \rho \mu \nu \lambda}\right],\left[c_{p}^{\mu \nu \rho \sigma \tau}\right] \in H_{5 m}\left(M ; \boldsymbol{Z}_{2}\right) .}
\end{aligned}
$$

Moreover, we denote by $B_{\imath}^{\lambda \pm}$ the ball such that $\partial B_{\imath}^{\lambda \pm}=S_{i}^{\lambda}=$ the hypersphere centered at the focal point $f_{i}^{\lambda}$ with radius $\cot ^{-1} \lambda\left(p_{i}\right)$, where $\boldsymbol{n}\left(p_{i}\right)$ (=the unit normal vector to $M$ at $p_{i}$ ) is the inner (outer, resp.) normal to $B_{\imath}^{\lambda+}\left(B_{2}^{\lambda-}\right.$, resp.). $B_{i}^{\mu \pm}, B_{\imath}^{\nu \pm}, B_{i}^{\rho \pm}, B_{\imath}^{\sigma \pm}$ and $B_{\imath}^{\tau \pm}$ are similarly defined.

Supposing $L_{2}^{\tau} \cap L_{4}^{\lambda}=\varnothing$, we may transform $M$ conformally so that $f_{2}^{\tau}$ and $f_{4}^{\lambda}$ are antipodal (see Figure 2). Let $x \in \gamma \backslash \widetilde{f_{2}^{\tau} p_{2}}$ be the point sufficiently near to $f_{2}^{\tau}$ 


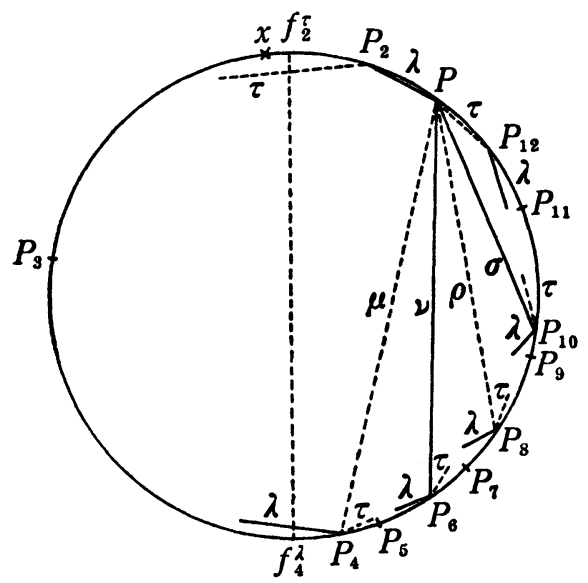

Fig. 2.

such that $d_{x}$ is a Morse function. We will lead a contradiction by showing that $d_{x}$ should have thirteen critical points. The minimum point of $d_{x}$ is in $B_{2}^{\tau-}$, and $p_{2}$ and $p_{1}$ are critical points of $d_{x}$ with index $m$, which correspond to cycles $c_{2}^{\tau}$ and $c_{2}^{\lambda}$, respectively. Next, we have $B_{12}^{\sigma+} \cap B_{2}^{\tau-} \neq \varnothing$, since the intersection number $S\left(c_{12}^{\sigma \rho \nu \mu \lambda}, c_{2}^{\tau}\right) \neq 0$, where $c_{12}^{\sigma o \nu \mu \lambda} \subset B_{12}^{\sigma+}$ and $c_{2}^{\tau} \subset B_{2}^{\tau-}$. This means that the critical point with index $2 m$ corresponding to $c_{12}^{\sigma \tau}$ should lie in $\left\{p \in M \mid d_{x}(p) \leqq d_{x}\left(p_{12}\right)\right\}$. In the same way, we can show that $B_{2}^{\mu-} \cap B_{12}^{\lambda+} \neq \varnothing$ and that the critical point with index $2 m$ corresponding to $c_{2}^{\mu \lambda}$ should lie in $\left\{p \in M \mid d_{x}(p) \leqq d_{x}\left(p_{12}\right)+2 \cot ^{-1} \lambda\left(p_{12}\right)\right\}$. Next, we have $B_{10}^{\rho+} \cap B_{12}^{\sigma-} \neq \varnothing$, because $S\left(c_{10}^{\rho \nu \mu \lambda}, c_{12}^{\sigma \tau}\right) \neq 0$, where $c_{10}^{\rho \nu \mu \lambda} \subset B_{10}^{\rho+}$ and $c_{12}^{\sigma \tau} \subset B_{12}^{\sigma-}$. Therefore, with $B_{12}^{\sigma+} \cap B_{2}^{\tau-} \neq \varnothing$, we know that the critical point with index $3 m$ corresponding to $c_{10}^{\text {oq }}$ should lie in $\left\{p \in M \mid d_{x}(\phi) \leqq d_{x}\left(p_{10}\right)\right\}$. In the same way, $B_{2}^{\nu-} \cap B_{12}^{\mu+} \neq \varnothing$ and $B_{12}^{\mu-} \cap B_{10}^{\lambda+} \neq \varnothing$ show that, the critical point with index $3 m$ corresponding to $c_{2}^{\nu \mu \lambda}$ should lie in $\left\{p \in M \mid d_{x}(p) \leqq d_{x}\left(p_{10}\right)+2 \cot ^{-1} \lambda\left(p_{10}\right)\right\}$. Note that $d_{x}\left(p_{10}\right)+2 \cot ^{-1} \lambda\left(p_{10}\right) \leqq d_{x}\left(p_{9}\right)$, since $\left(B_{10}^{\lambda+}\right)^{\circ} \cap M=\varnothing$. So all seven critical points above lie in $\left\{p \in M \mid d_{x}(p)<d_{x}\left(p_{8}\right)\right\}$.

On the other hand, $d_{-x}$ should have the minimum point $p_{4}$, critical points with index $m$ corresponding to $c_{4}^{\lambda}$ and $c_{4}^{\tau}$ in $\left\{p \in M \mid d_{-x}(p) \leqq d_{-x}\left(p_{4}\right)+2 \cot ^{-1}\left(-\tau\left(p_{4}\right)\right)\right\}$, critical points of index $2 m$ corresponding to $c_{6}^{\mu \lambda}$ and $c_{4}^{\sigma \tau}$ in $\left\{p \in M \mid d_{-x}(p) \leqq d_{-x}\left(p_{6}\right)\right.$ $\left.+2 \cot ^{-1}\left(-\tau\left(p_{6}\right)\right)\right\}$. Thus these five critical points lie in $\left\{p \in M \mid d_{x}(p)>d_{x}\left(p_{8}\right)\right\}$. Now, since $p_{8}$ is another critical point of $d_{x}, d_{x}$ should have thirteen critical points on $\gamma$, a contradiction.

Thus we get $L_{2}^{\tau} \cap L_{4}^{\lambda}=p_{3}$, and similarly $L_{4}^{\tau} \cap L_{6}^{\lambda}=p_{5}, L_{6}^{\tau} \cap L_{8}^{\lambda}=p_{7}, L_{8}^{\tau} \cap L_{10}^{\lambda}=p_{9}$ and $L_{10}^{\tau} \cap L_{12}^{\lambda}=p_{11}$. Further argument using tautness shows that these twelve points are connected each other by certain leaves as in Figure $1 . \quad$ q.e.d.

LEMMA 4. By a Lie transformation $A_{3} \in O(n+1,2)$, we can transform $M$ so that $p_{1}, p_{2}, \cdots, p_{12}$ are the vertices of a regular dodecagon. 
Proof. The conformal transformation which takes $L_{1}^{\lambda}$ and $L_{7}^{\lambda}$ to the antipodal position is easily found. Now, preserving this relation, we can find a Lie transformation such that $\tau\left(p_{1}\right)=\tau\left(p_{2}\right)$ (see $\S 7$ of [1]). Then the constantness of cross ratios shows that all principal curvatures at $p_{1}$ and $p_{2}$ coincide. Moreover, preserving this relation, we can find another Lie transformation such that $\tau\left(p_{3}\right)=\tau\left(p_{6}\right)$ (see Prop. 8.1 of [1], especially the footnote given in its proof). Thus each of $\mu, \nu, \tau$ takes the same value at $p_{3}$ and $p_{6}$, and so do $\lambda, \rho$ and $\sigma$ by the assumption. Therefore we get Figure 3 where $\theta_{1}=\cot ^{-1} \lambda\left(p_{1}\right)$,

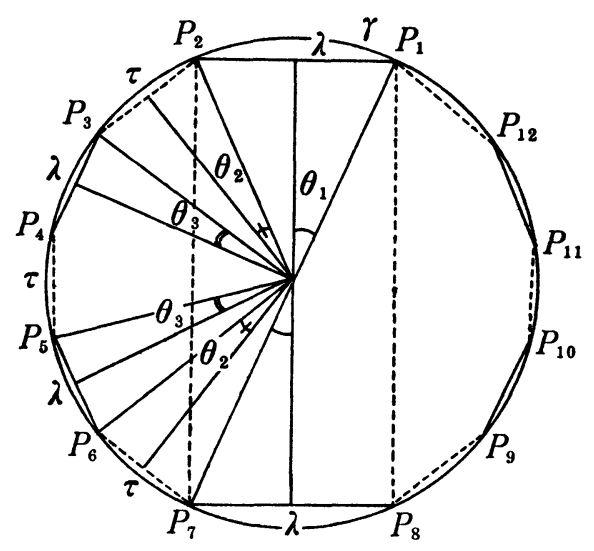

Fig. 3.

$\theta=-\cot ^{-1} \tau\left(p_{2}\right)$ and $\theta_{3}=\cot ^{-1} \lambda\left(p_{3}\right)$. Let $z_{2}$ be the complex number corresponding to $p_{i}$ where we may assume $z_{1}=1$. Put $\Psi=\frac{(\lambda-\nu)(\lambda-\tau)}{(\lambda-\tau)(\mu-\nu)}$. Then we have by Lemma 6.8 of [1],

$$
\begin{aligned}
& \Psi\left(p_{2}\right)=\left[z_{1}, z_{11} ; z_{9}, z_{3}\right] \\
& \Psi\left(p_{10}\right)=\left[z_{9}, z_{7} ; z_{5}, z_{11}\right] .
\end{aligned}
$$

Since $z_{7}=-z_{1}=-1, z_{9}=-z_{3}$ and $z_{11}=-z_{5}, \Psi\left(p_{2}\right)=\Psi\left(p_{10}\right)$ implies

i.e.

$$
1+z_{3} z_{5}=0
$$

Thus we obtain

$$
2\left(\theta_{1}+\theta_{2}\right)+\left\{\pi-2\left(\theta_{2}+\theta_{3}\right)\right\}=\pi .
$$

$$
\theta_{1}=\theta_{3} .
$$

Therefore, we get through a parallel transformation that

$$
\theta_{1}=\theta_{2}=\theta_{3}=\frac{\pi}{12} \text {. }
$$


Proposition 5. A Lie image of an isoparametric hypersurface satisfies (ii).

Proof. Since the relation (ii) is preserved by Lie transformations, we show that an isoparametric hypersurface $N$ satisfies (ii). Note that at any point $p_{1} \in N$, the intersection of the normal geodesic $\gamma$ at $p_{1}$ and $N$ makes a regular dodecagon as in Figure 4 after a suitable parallel transformation. We will show that for any $q \in L_{1}^{\lambda}, L_{q}^{\mu} \cap L_{4}^{\nu} \neq \varnothing$. In fact, it is an easy consequence of $S\left(c_{q}^{\mu \lambda}, c_{4}^{\nu \rho \sigma \tau}\right) \neq 0$ and $\mu(q) \equiv \mu\left(p_{1}\right)$. Other cases follow similarly. q.e.d.

Now, consider sufficiency. Under the condition (i), we could transform the original hypersurface $M$ to a hypersurface $\tilde{M}$ satisfying the relation in Figure 4 at the image point of some fixed point of $M$. Now, when $\tilde{M}$ satisfies (ii), for a $\lambda$-leaf $L^{\lambda}$, denote by $L_{\lambda}^{\nu}$ the $\nu$-leaf satisfying $L_{q}^{\mu} \cap L_{\lambda}^{\nu} \neq \varnothing, q \in L^{\lambda} . \quad L_{\lambda}^{\sigma}$ is defined

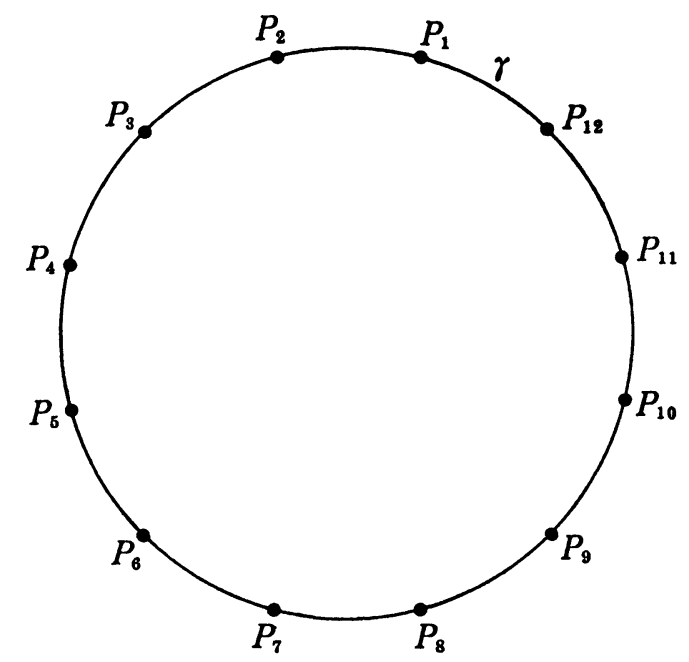

Fig. 4.

similarly. Then by the same argument as in the proof of Lemma 9.2 of [1], we can show $L_{\lambda}^{\nu}=L_{4}^{\nu}$ and $L_{\lambda}^{\sigma}=L_{6}^{\sigma}$. Thus it is easy to see that $T L_{1}^{\lambda}=T L_{4}^{\nu}=T L_{6}^{\sigma}$, where "=" means "be parallel to" with respect to the connection of $S^{n}$ (see the proof of Proposition 9.3 of [1]). Note that these facts hold also for $L_{3}^{\lambda}, L_{5}^{\lambda}, L_{7}^{\lambda}$, $L_{9}^{\lambda}$ and $L_{11}^{\lambda}$. Now, we show:

LEMMA 6. The parallel families of tangent spaces of leaves are $\left\{T L_{2}^{\lambda}, T L_{3}^{\sigma}\right.$, $\left.T L_{4}^{\nu}, T L_{5}^{\nu}, T L_{6}^{\sigma}, T L_{7}^{\lambda}\right\}, \quad\left\{T L_{3}^{\tau}, T L_{4}^{\mu}, T L_{5}^{\rho}, T L_{6}^{\rho}, T L_{7}^{\mu}, T L_{8}^{\tau}\right\}, \quad\left\{T L_{4}^{\lambda}, T L_{5}^{\sigma}, T L_{6}^{\nu}\right.$, $\left.T L_{7}^{\nu}, T L_{8}^{\sigma}, T L_{9}^{\lambda}\right\}, \quad\left\{T L_{5}^{\tau}, T L_{6}^{\mu}, T L_{7}^{\rho}, T L_{8}^{\rho}, T L_{9}^{\mu}, T L_{10}^{\tau}\right\}, \quad\left\{T L_{6}^{\lambda}, T L_{7}^{\sigma}, T L_{8}^{\nu}, T L_{y}^{\nu}\right.$, $\left.T L_{10}^{\sigma}, T L_{11}^{\lambda}\right\}$ and $\left\{T L_{7}^{\tau}, T L_{8}^{\mu}, T L_{9}^{\rho}, T L_{10}^{\rho}, T L_{11}^{\mu}, T L_{12}^{\tau}\right\}$. 
Proof. Put $T L_{1}^{\lambda}=U, T L_{1}^{\mu}=V, T L_{1}^{\nu}=W, T L_{1}^{\rho}=X, T L_{1}^{\sigma}=Y, \quad T L_{1}^{\tau}=Z$, $T L_{7}^{\lambda}=U_{1}, T L_{7}^{\mu}=V_{1}, T L_{7}^{\nu}=W_{1}, T L_{7}^{\rho}=X_{1}, T L_{7}^{\sigma}=Y_{1}, T L_{7}^{\tau}=Z_{1}, T L_{2}^{\mu}=V_{2}, T L_{2}^{\tau}=Z_{2}$, $T L_{8}^{\mu}=V_{3}$ and $T L_{8}^{\tau}=Z_{3}$. From above fact, it follows that $T L_{4}^{\nu}=T L_{6}^{\sigma}=U$, $T L_{3}^{\lambda}=T L_{8}^{\sigma}=W, T L_{5}^{\lambda}=T L_{3}^{\nu}=Y, T L_{3}^{\sigma}=T L_{5}^{\nu}=U_{1}, T L_{2}^{\sigma}=T L_{9}^{\lambda}=W_{1}$ and $T L_{2}^{\nu}=T L_{11}^{\lambda}$ $=Y_{1}$. Thus, we have

$$
\begin{aligned}
& U_{1} \oplus V_{3} \oplus Z_{3}=U \oplus V \oplus Z, \\
& U_{1} \oplus V_{1} \oplus Z_{1}=U \oplus V_{2} \oplus Z_{2},
\end{aligned}
$$

since $T_{1} M=T_{8} M$ and $T_{2} M=T_{7} M$. Noting that $U_{1} \perp Z$ at $p_{12}$ and $U_{1} \perp Z_{2}$ at $p_{3}$, we get

$$
U_{1} \subset(U \oplus V) \cap\left(U \oplus V_{2}\right),
$$

but since $L_{1}^{\mu} \cap L_{2}^{\mu}=\varnothing$, we have $V \cap V_{2}=\{0\}$, i. e. $U_{1}=U$. By the same argument at $p_{3}$ and $p_{5}$, we get $W_{1}=W$ and $Y_{1}=Y$.

Now, consider the hexagon with vertices $p_{1}, p_{4}, p_{5}, p_{8}, p_{9}, p_{12}$. At each vertex, just note $\mu, \rho, \tau$-leaves. Then the total tangent space of these three leaves is equal to $V \oplus X \oplus Z$ at each vertex. So by an easy argument as above, using that $\rho$-leaves never intersect each other, we get $T L_{5}^{\rho}=T L_{8}^{\tau}=V, T L_{4}^{\tau}=$ $T L_{9}^{\mu}=X$ and $T L_{4}^{\rho}=T L_{5}^{\mu}=Z$.

Claim. On $L_{1}^{\tau}, \mu$ and $\rho$ are constant and their leaves are totally geodesic.

Since $T L_{4}^{\rho}=T L_{5}^{\mu}=Z$, the normal geodesic at $q \in L_{1}^{\tau}$ cuts $M$ as in Figure 5 in which the definition of $q_{1}$ and $q_{2}$ is given. Then, $S\left(c_{q_{1}}^{\mu \nu \rho \sigma \tau}, c_{q}^{\lambda}\right) \neq 0$ implies $\mu\left(q_{1}\right) \geqq \frac{3 \pi}{12}$, and $S\left(c_{q_{1}}^{\mu \lambda}, c_{q}^{\nu \rho \sigma \tau}\right) \neq 0$ implies $\mu\left(q_{1}\right) \leqq \frac{3 \pi}{12}$. Thus we have $\mu\left(q_{1}\right)=\frac{3 \pi}{12}$,

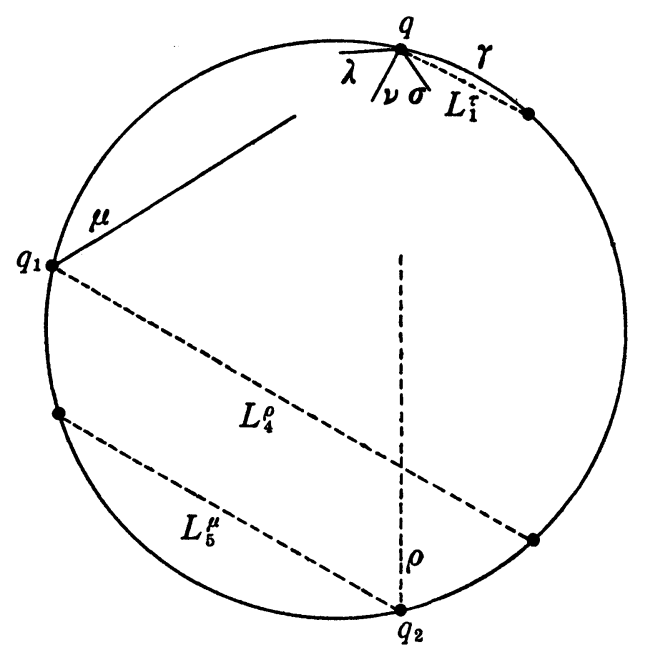

Fig. 5 . 
from which follows $c_{q}^{\lambda} \cap c_{q_{1}}^{\mu \nu \rho \sigma \tau} \subset B_{q}^{\lambda+} \cap B_{q_{1}}^{\mu-}=q$ so that $q \in L_{q_{1}}^{\mu}$ and $L_{q}^{\mu}=L_{q_{1}}^{\mu}$ is totally geodesic by Remark 6.2 of [1]. In the same way, $S\left(c_{q_{2}}^{\rho \nu \mu \lambda}, c_{q}^{\sigma \tau}\right) \neq 0$ and $S\left(c_{q_{2}}^{\rho \sigma \tau}, c_{q}^{\nu \mu \lambda}\right) \neq 0$ imply $\rho\left(q_{2}\right)=\cot \frac{5 \pi}{12}$, and $L_{q}^{\rho}=L_{q_{2}}^{\rho}$ is totally geodesic.

Now, we get from the assumption (i) that all principal curvatures are constant on $L_{1}^{\tau}$. Or, more strongly:

Claim. All leaves through a point of $L_{1}^{\tau}$ is totally geodesic.

This is because, we have $\Lambda_{\beta \beta}^{\alpha} \equiv 0$ for $\alpha \notin[\beta]$, where $\beta=f, \gamma, x$ on $L_{1}^{\tau}$, then Lemma 1 implies all $\Lambda_{\beta \beta}^{\alpha} \equiv 0$ on $L_{1}^{\tau}$ for any $\alpha \notin[\beta]$.

Thus we must have $T L_{2}^{\mu}=T L_{3}^{\rho}=T L_{6}^{\tau}=Z$, and similarly, $T L_{2}^{\tau}=T L_{6}^{\rho}=T L_{7}^{\mu}$ $=V, T L_{3}^{\mu}=T L_{2}^{\rho}=T L_{10}^{\tau}=X$ (see Remark 6.2 of [1]). This proves Lemma 6 .

Proof of sufficiency. Similar to the proof of Theorem II in [1].

q.e.d.

\section{REFERENCES}

[1] MrYaOKA, R.: Dupin hypersurfaces and a Lie invariant. Kodai Math. J. 12, 228-256 (1989).

[2] Münzner, H.F.: Isoparametrische Hyperflächen in Sphären I. Math. Ann. 251, 57-71 (1980).

[3] Thorbergsson, G.: Dupin hypersurfaces. Bull. London Math. Soc. 15, 493-498 (1983).

Department of Mathematics

TOKYO INSTITUTE OF TECHNOLOGY

Ohokayama, Meguroku 